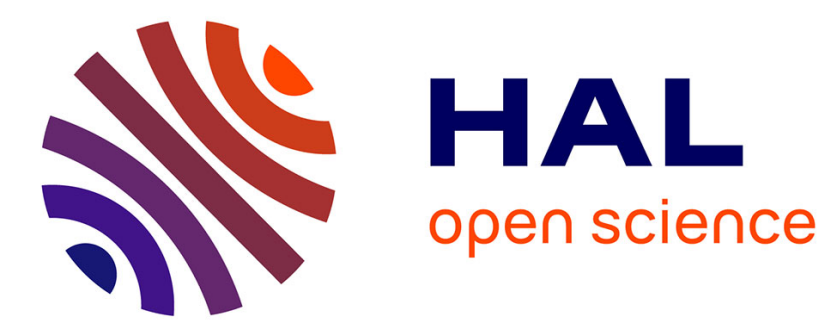

\title{
Absolute and convective instabilities of fluidized beds
}

Maxime Nicolas, Jean-Marc Chomaz, Elisabeth Guazzelli

\section{To cite this version:}

Maxime Nicolas, Jean-Marc Chomaz, Elisabeth Guazzelli. Absolute and convective instabilities of fluidized beds. Physics of Fluids, 1994, 6 (12), pp.3936-3944. 10.1063/1.868446 . hal-01432852

\section{HAL Id: hal-01432852 \\ https://hal-amu.archives-ouvertes.fr/hal-01432852}

Submitted on 12 Jan 2017

HAL is a multi-disciplinary open access archive for the deposit and dissemination of scientific research documents, whether they are published or not. The documents may come from teaching and research institutions in France or abroad, or from public or private research centers.
L'archive ouverte pluridisciplinaire $\mathbf{H A L}$, est destinée au dépôt et à la diffusion de documents scientifiques de niveau recherche, publiés ou non, émanant des établissements d'enseignement et de recherche français ou étrangers, des laboratoires publics ou privés. 


\title{
Absolute and convective instabilities of fluidized beds
}

\author{
Maxime Nicolas \\ Laboratoire de Physique et Mécanique des Milieux Hétèrogènes, URA 857 au CNRS, ESPCI, \\ 10 rue Vauquelin, 75231 Paris Cedex 05, France \\ Jean-Marc Chomaz \\ LadHyX, Ecole Polytechnique, URA 317 au CNRS, 91128 Palaiseau Cedex, France \\ Elisabeth Guazzelli \\ Laboratoire de Physique et Mécanique des Milieux Hétèrogènes, URA 857 au CNRS, ESPCI, \\ 10 rue Vauquelin, 75231 Paris Cedex 05, France
}

(Received 31 May 1994; accepted 2 August 1994)

The stability of a fluidized bed is investigated with respect to spatially growing disturbances. A general linearized model is derived from the theories of Anderson and Jackson and of Batchelor. The absolute and convective nature of the instability is analyzed using the mathematical framework of the open flow linear stability theory. The results of the analysis provide the domains of absolute and convective instabilities. (C) 1994 American Institute of Physics.

\section{INTRODUCTION}

A fluidized bed consists of a suspension of solid particles supported against gravity by an upward flowing fluid. ${ }^{1} \mathrm{~A}$ fluidized bed can be considered as an ensemble of sedimenting particles referred to different reference frames and hence as a stationary sedimenting suspension. Under some conditions, stable expansions have been found experimentally over a finite interval of flow rates beyond the minimum flow rate for fluidization. ${ }^{2,3}$ However, the ideal, uniform, and homogeneous state of fluidization is rarely realized in practice. Instead, fluidized beds exhibit different regimes of complex motion depending upon the flow rate of the injected fluid. Gas-fluidized beds are usually very unstable and rapidly attain a turbulent regime traversed by rising bubbles, i.e., regions essentially devoid of particles which rise through the bed when the flow rate is increased (see, for instance, the reviews of Clift and Grace ${ }^{4}$ and Davidson et al. ${ }^{5}$ ). Liquidfluidized beds present voidage instability waves. ${ }^{6-8}$ This first wayy instability destabilizes and leads to secondary instabilities such as transversal structures when the flow rate is increased. ${ }^{9,10}$ Further increase of the flow rate leads to turbulent and bubbly regimes. ${ }^{8}$

Although fluidized beds have been studied and practically used for a long time, the underlying physical mechanisms are still poorly understood. One of the main difficulties lies in the complete understanding of particle-particle and particle-fluid interactions. There is still no general consensus regarding the governing dynamical equations of fluidized beds. Schematically, two different approaches have been developed. Over the past two decades, two-phase continuum modeling has been applied to this problem (see, for instance, the reviews of Jackson ${ }^{11}$ and Homsy ${ }^{12}$ ). Most of these models considered the linearized hydrodynamic stability of an uniform and infinite fluidized bed and the hydrodynamics of an isolated idealized bubble. More recently, a new theory of the instability of a uniform, infinite, and onedimensional fluidized bed was proposed by Batchelor. ${ }^{13}$ The governing equations have been established from a physical picture of the system as a suspension of particles interacting with a flowing fluid.

Since the occurrence of bubbles is an important and practical feature of fluidized beds, numerous studies have been devoted to their investigation. However, the origin of the bubbly regime is still unknown. Experimental observations suggest that bubble formation is linked to the secondary instabilities of the voidage instability waves. ${ }^{7,9}$ A recent conjecture is that bubbles originate from a gravitational overturning instability caused by the first wavy disturbance. ${ }^{10,13,14}$ It is, therefore, relevant to investigate the different instabilities of a fluidized bed and the transition toward the turbulent and bubbly regimes. The investigation of the first wavy instability, which is an interesting problem in itself, is the first step toward the understanding of bubble formation.

Experimental studies of the first wavy instability have been conducted in liquid-fluidized beds. The measured disturbances have been shown to grow exponentially upwards along the bed and eventually to lead to a saturated finite amplitude. ${ }^{6,7}$ Although a dominant instability mode was clearly evidenced, the power spectrum of the voidage fluctuations was found to be very broad. These experimental findings suggest that the first wavy instability is convective in nature and that the fluidized bed behaves as a spatial noise amplifier where any small perturbation created at the entrance of the bed is amplified along it. Fluidized beds belong, indeed, to the open flow class where fluid elements continuously enter and leave the experimental system. Therefore, the theory of instability waves in fluidized beds should take into account the open flow features, i.e., the spatial origin of the flow and the mean advection.

Temporally growing disturbances have only been considered in theoretical work. ${ }^{11-13,15-22}$ This temporal description has provided valuable quantitative information such as the stability condition, the dominant wavelength, and the temporal growth rate. However, when theoretical predictions were compared with experimental results, the spatial growth rate was approximated as the temporal growth rate multiplied by the mean velocity. ${ }^{6,7}$ Advances in open flow stability 
theory should provide an accurate description of the spatial growth of the wave instability (see, for instance, the reviews of Briggs, ${ }^{23}$ Bers, ${ }^{24}$ and Huerre and Monkewitz ${ }^{25}$ ). It should also yield information on the convective or absolute nature of the instability.

The specific purpose of this paper is to investigate the stability of a fluidized bed with respect to spatially growing disturbances and to look for conditions under which a transition between convective and absolute instabilities can be identified. To accomplish this goal, we consider in Sec. II a general linearized model derived from the theories of Anderson and Jackson, ${ }^{18,26}$ and of Batchelor. ${ }^{13}$ Linear stability is investigated in Sec. III using the mathematical framework of the open flow theory. The marginal stability condition as well as the convective/absolute instability transition condition are determined. The conditions for convective and absolute instabilities are put into the physical parameter framework in Sec. IV and conclusions are drawn in Sec. V.

\section{THEORETICAL MODEL}

In this section, we consider the general form of the linearized equation for small perturbations of the particle volume fraction. The fluidized bed is assumed to be onedimensional and unbounded. The fluidizing fluid may be either a liquid or a gas, with a density $\rho_{f}$ and with a viscosity $\mu_{f}$. The solid particles are supposed to be non-Brownian monodisperse spheres, with diameter $d_{s}$ and density $\rho_{s}$.

Two-phase flow modeling uses equations of motion based on a continuum picture of the suspension. The usual procedure is to write the continuity and momentum equations for each of the two phases of the system, the particles, and the fluid. At this stage, these equations formally contain a force representing the interaction between the two phases and the stress tensors associated with the fluid and particle phases. In order to close the equations, it is necessary to postulate expressions for these terms. Since this can only be done on a heuristic basis, it is here that the greatest differences arise among equations proposed in the literature. For the purpose of the present work, the model of Anderson and Jackson ${ }^{11,18,26}$ has been chosen. In this model, the force exerted by the fluid on the particles contains two terms. The first term is a drag force depending on the particle volume fraction $\phi$ and the relative velocity of the two phases. The second term represents virtual mass effects and is proportional to the relative acceleration of the phases. The virtual mass coefficient, whose value would be $1 / 2$ for an isolated sphere, is a function of the particle volume fraction $C(\phi)$. The stress tensors associated with the fluid and the particle phases are independent and both have the Newtonian fluid form. Although the pressure, the bulk, and shear viscosities have a clear meaning for the fluid phase, the pressure $p^{s}$ and the bulk and shear viscosities $\lambda^{s}$ and $\mu^{s}$, respectively, for the particle phase cannot be easily estimated or measured. Nevertheless, these governing equations have been used to write a linearized equation for the small perturbation of the particle volume fraction $\phi_{1}$ [Eq. (19) in Anderson and Jackson ${ }^{18}$ ].

Batchelor's approach is rather different. ${ }^{13} \mathrm{He}$ considers the general form of the equations that govern the mean motion of the particles in the vertical direction. He assumes that the particles and fluid are incompressible and that there is no acceleration of the mixture as a whole. The velocity of the mixture is therefore space and time independent. The governing equations express the conservation of the particle and momentum. In the approximate form for small departures from uniformity, the rate of change of particle momentum is equal to four terms [Eq. (3.10) of Batchelor ${ }^{13}$ ]. The first term describes the acceleration reaction of the particles. It is again necessary at this stage to introduce the virtual mass coefficient $C(\phi)$. The second term is the fluid drag-weight term. The third term can be identified as a viscous term. This term contains a coefficient $\eta$ which has the dimension of a diffusivity (or a kinematic viscosity) and is termed as the particle viscosity. The last term is proportional to the spatial gradient of the particle volume fraction. The coefficient $Q$ of the gradient can be interpreted as an effective bulk modulus of elasticity of the particle configuration divided by the particle mass by unit volume of the mixture. It represents the sum of two different effects, one arising from the transfer of particle momentum by velocity fluctuations analogous to the Reynolds stress in turbulence and the other being the hydrodynamic particle diffusion down a concentration gradient. This gradient diffusion which arises from random fluctuations in the particle velocity is estimated to provide the largest contribution to $Q$ and to be responsible for the stability of the bed. The two governing equations are then written for small perturbations as Eqs. (3.13) and (3.14) in Ref. 13. It is important to mention that the reference frame used by Batchelor is the usual reference frame of sedimentation where the mean velocity of the mixture is zero. For the completion of the present spatial stability analysis, the appropriate reference frame is that of the fluidized bed where the mean velocity of the particles is zero. The linearized equations of Batchelor for small perturbations have thus been written in this latter reference frame.

To complete the presentation of the two models, it is important to discuss the origins of the stabilizing and destabilizing mechanisms in fluidized beds. Particle inertia was recognized early as the destabilizing mechanism. ${ }^{27}$ The effect of inertia produces some delay in the adjustment of particle velocity to a change in the local concentration and hence can promote the growth of the wave. Conversely, the physical origin of the stabilizing mechanism is still controversial. Schematically, two physical origins for stability have been invoked, one being the solid contact forces between particles and the other being the hydrodynamic gradient diffusion of the particles due to particle velocity fluctuations. In the work of Anderson and Jackson, ${ }^{18}$ the bed was always found unstable. Later, it was recognized that the bed could be stabilized for a sufficiently large value of $d p^{s} / d \phi{ }^{19}$ Since $d p^{s} / d \phi$ measures the rate of change of particle pressure with concentration, it represents a bulk modulus of elasticity of the particle phase. In Batchelor's model, ${ }^{13}$ the elastic behavior of the particle configuration is represented by the parameter $Q$. The elasticity of the particles hinders the growth of the waves by homogenizing the concentration gradients and can cause the bed stability.

Both models give the same form for the linearized equation for small perturbations of the particle volume fraction 
$\phi_{1}$. It is convenient to recast this equation into the form of a wave hierarchy equation, ${ }^{2,28,29}$ in the fluidized bed reference frame, and with the vertical axis $O x$, oriented upward and with the time $t$ :

$$
\begin{gathered}
\frac{\partial \phi_{1}}{\partial t}+c_{0} \frac{\partial \phi_{1}}{\partial x}+\tau\left(\frac{\partial}{\partial t}+c_{1} \frac{\partial}{\partial x}\right)\left(\frac{\partial}{\partial t}+c_{2} \frac{\partial}{\partial x}\right) \phi_{1} \\
=\nu \tau \frac{\partial}{\partial t} \frac{\partial^{2} \phi_{1}}{\partial x^{2}} .
\end{gathered}
$$

The coefficients $\tau, c_{1}, c_{2}$, and $\nu$ differ for the two theories and are given in the Appendix. The waves of different orders are displayed by the factored operators on the left-hand side of Eq. (1). The lowest order wave is the linearized form of the kinematic concentration wave found by Kynch to describe the propagation of the front separating the clear fluid and the suspension in sedimentation. ${ }^{30}$ The kinematic wave speed is identical in both theories and is equal to $c_{0}=-[\phi(d U / d \phi)]_{0}$ where $U(\phi)$ is the mean velocity of the mixture, which is usually called the superficial velocity, in the homogeneous state labeled 0 . A widely used correlation, called Richardson and Zaki correlation, gives the relationship between $U$ and $\phi$ as $U(\phi)=U_{0}(1-\phi)^{n}$, where the index $n$ varies monotonically with the particle Reynolds number $\left(\operatorname{Re}=d_{s} U_{0} \rho_{f} / \mu_{f}\right)$ and $U_{0}$ is the Stokes velocity of a single sphere for small Reynolds number. ${ }^{31}$ The higher order waves are usually termed dynamic waves. In the absence of virtual mass effects as in gas-fluidized beds, the dynamic waves speeds $c_{1}$ and $c_{2}$ reduce to $\pm Q^{1 / 2}$ in Batchelor's model and the physical origin of the waves lies in the physical processes represented by $Q$. Otherwise, the physical processes involved are less clear. The term on the right-hand side of Eq. (1) can be interpreted as a higher order viscous term. Although the wave-hierarchy interpretation of fluidized bed instabilities may not be completely relevant, Eq. (1) has been used to perform the stability analysis because of its compactness.

\section{INSTABILITY PROPERTIES OF THE LINEARIZED MODEL}

Equation (1) contains five coefficients. This number can be reduced to three by making the variables dimensionless. We therefore put $T=t / \tau$ and $X=x /\left(\tau / c_{0}\right)$. We also consider a small perturbation of the particle volume fraction that varies as $\exp [i(K X-\Omega T)]$. In the $(X, T)$ variables, the dispersion relation can be written as

$$
(\Omega-K c)(\Omega+K c f)+i(\Omega-K)+i a \Omega K^{2}=0,
$$

where $c=c_{1} / c_{0}, f=-c_{2} / c_{1}$ which can be termed a dissymmetry parameter, and $a=\nu /\left(\tau c_{0}^{2}\right)$ which represents the inverse of a Reynolds number based on a velocity scale $c_{0}$ and a length scale $\tau c_{0}$.

\section{A. Temporal stability analysis}

Temporal stability analysis relates to the time evolution of a spatially homogeneous wave defined by a real wave number, $K_{r}$, but a complex frequency, $\Omega=\Omega_{r}+i \Omega_{i}$. Hence $\Omega_{i}>0$ gives the temporal growth rate of unstable disturbances. Since temporally growing disturbances have been considered in numerous theoretical studies, ${ }^{11-13,15-22}$ the major findings of the analysis are briefly presented in this section.

The disturbances of greatest relevance to stability questions are those of long wavelength and therefore the stability condition does not depend on the coefficient $a$. At marginal stability, the most unstable wave number is $K_{r}=0$ and the stability criterion can be written as ${ }^{2,28,29}$

$$
-f c<1<c .
$$

This stability criterion simply states that the kinematic wave speed lies between the two dynamic wave speeds and is similar to that derived by Wallis. ${ }^{32}$ This stability criterion is also equivalent to that of Batchelor ${ }^{13}$ [his Eq. (4.12)] which in the present formulation is $N_{m}=\left[f c^{2}+c(1-f)\right]^{-1}<1$.

The dependence of the temporal growth rate $\Omega_{i}$ on $K_{r}$ can also be investigated. The temporal branch relevant for stability question is shown in Fig. 1 for $c=1.2$ (a1), 0.48 (a2), and 0.44 (a3) and for $a=1$ and $f=1$. For $c>1$, the fluidized bed is stable and the temporal branch lies under the real $K$ axis. For $c<1$, the range of wave numbers for which $\Omega_{i}>0$ is $0<K_{r}<K_{r}^{n}$, where $K=0$ and $K_{r}^{n}$ are the neutrally unstable wave numbers. This later neutrally unstable wave number is given by $K_{r}^{n}=[(1-c) / a c]^{1 / 2}$ which corresponds to a frequency $\Omega_{r}^{n}=c K_{r}^{n}$. The most temporally unstable wave number $K_{r}^{t m}$ corresponds to the maximum temporal growth $\Omega_{i}^{t m}$ and the most temporally unstable frequency $\Omega_{r}^{t m}$.

\section{B. Spatial stability analysis}

Spatial stability analysis considers the response of the flow to a localized harmonic forcing with steady amplitude. This type of analysis is particularly well suited for the noise forcing problem at the spatial origin of fluidized beds. In contrast to temporal stability analysis, the wave frequency is kept real, $\Omega_{r}$, while the wave number is complex, $K=K_{r}+i K_{i}$. Hence $-K_{i}>0$ only gives unstable disturbances propagating upwards. ${ }^{33}$

The two spatial branches are plotted in the $\left(K_{r},-K_{i}\right)$ plane in Fig. 1 for the same values of the coefficients $a, f$, and $c$ as those used for the temporal branch. For $c>1$, the spatial branches do not cross the real axis but lie on either side of it [Fig. 1(b1)]. Since the flow is stable, the response to forcing is damped. The branch in the upper $K$ domain $\left(K^{+}\right)$corresponds to energy propagation to the right-hand side of the harmonic source (located at $X=0$ ) toward $+\infty$ while the branch in the lower $K$ domain $\left(K^{-}\right)$corresponds to propagation toward $-\infty$ [Fig. 1(c1)]. The instability is associated with the crossing of the real $K$ axis by one spatial branch when $c<1$ [Fig. 1(b2)]. Since no topological change occurs for the spatial branches at the instability transition, by using a continuity argument it can be shown that each spatial branch is associated with the same propagation direction as in the stable case [Fig. 1(c2)]. The wave propagating toward $+\infty$ is then amplified for $0<K_{r}<K_{r}^{n}$, while the wave propagating to $-\infty$ is still damped. The unstable spatial branch presents a maximum at $-K_{i}^{s m}$ which corresponds to a wave number $K_{r}^{s m}$ and a frequency $\Omega_{r}^{s m}$. This continuity argument becomes unvalid when the two spatial branches issuing from the upper and lower real $K$ half-plane $\left(K^{+}\right.$and $\left.K^{-}\right)$collide 

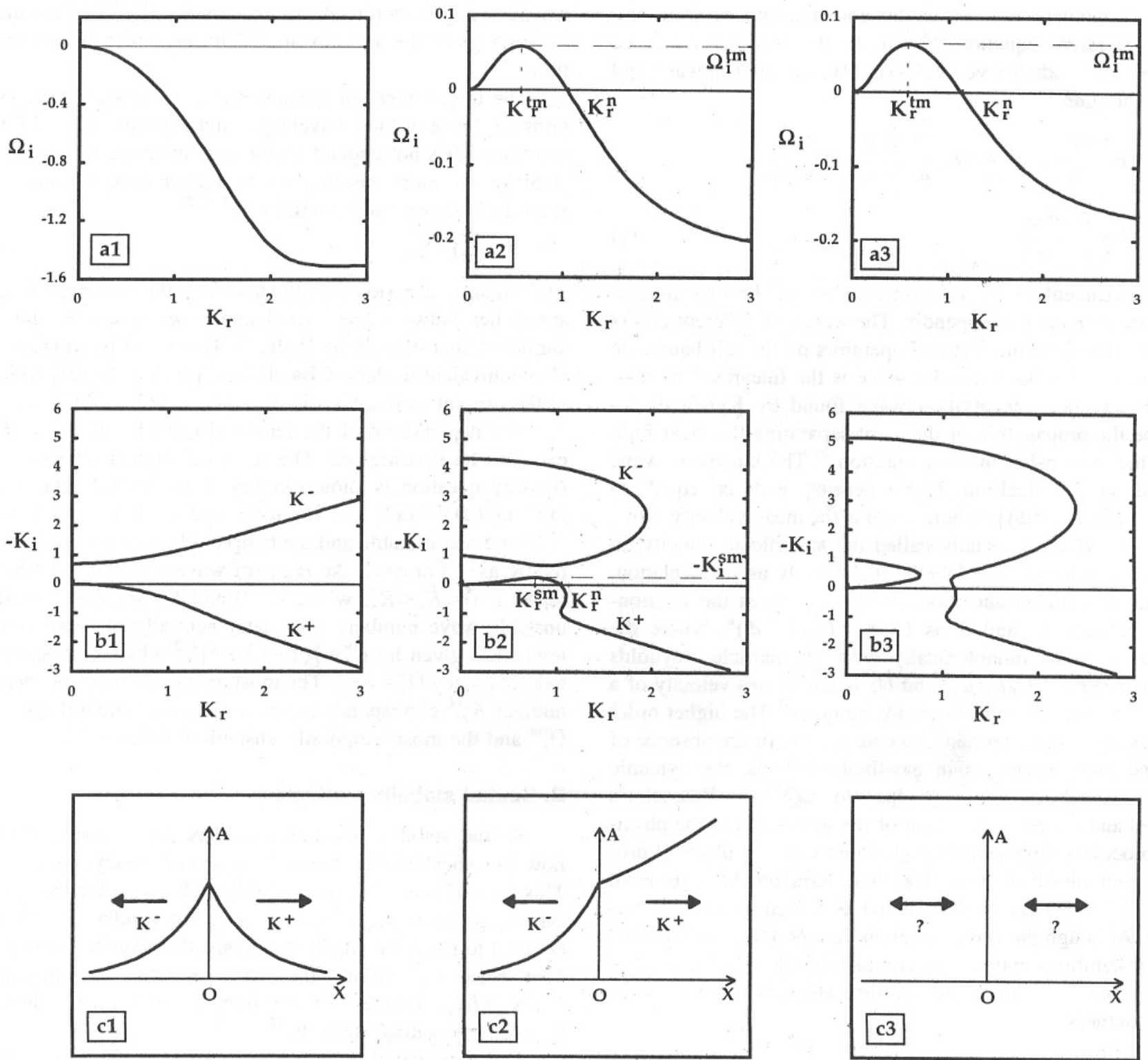

FIG. 1. Temporal branches (a), spatial branches (b), and responses to a forcing localized in space and harmonic in time (c): Stable case, $c=1.2$ (1), convectively unstable case, $c=0.48$ (2), and absolutely unstable case, $c=0.44$. For all cases, $a=1$ and $f=1$.

[Fig. 1(b3)]. Since no propagation direction can be associated with the spatial branches, the response to the source cannot be defined [Fig. 1(c3)]. It should be noticed that no topological change is observed for the temporal branches [Fig. 1(a3)]. This transition occurs when the saddle point $\left(\Omega^{0}, K^{0}\right)$ of the dispersion relation $\left[\Omega^{0}=\Omega\left(K^{0}\right)\right.$, $\left.d \Omega / d K\left(K^{0}\right)=0\right]$ crosses the $\Omega$ real plane $\left(\Omega_{i}^{0}=0\right)$.

As a summary of the spatial analysis, while the maximum imaginary part of the temporal mode $\Omega_{i}^{t m}$ defines through its sign the stability of the flow, another quantity, termed the absolute growth rate $\Omega_{i}^{0}$, determines the ability to define the response to a localized source. When $\Omega_{i}^{0}<0$, this response is defined and the flow is said to be convectively unstable. When $\Omega_{i}^{0}>0$, the instability is absolute and the response to forcing cannot be defined. The disturbances will grow both temporally and spatially at the onset of absolute instability.

\section{Convective/absolute transition}

In the above section, two classes of spatially evolving flow have been defined. Convectively unstable flows behave as spatial amplifiers of the incoming perturbations whereas absolutely unstable flows have an intrinsic behavior since they are insensitive to localized forcing. The change in the behavior of open flows when the flow becomes absolutely unstable has been demonstrated in numerous experiments and numerical simulations (see, for instance, the review of Huerre and Monkewitz ${ }^{25}$ ).

In the case of the present linearized model of fluidized 


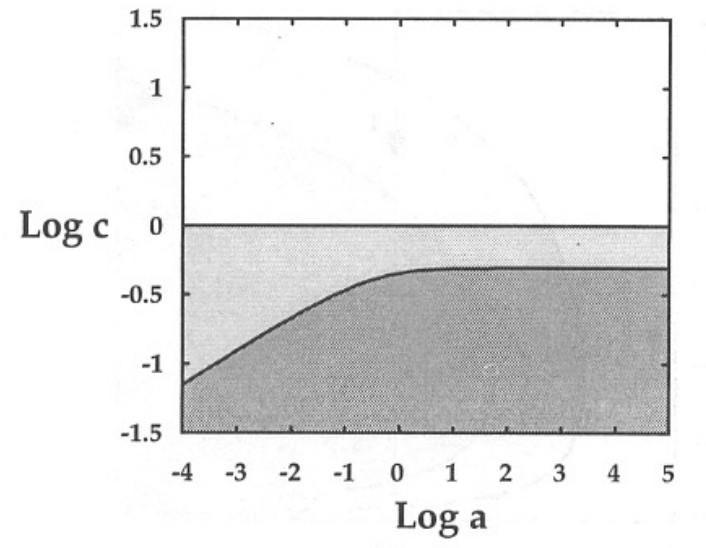

(a)
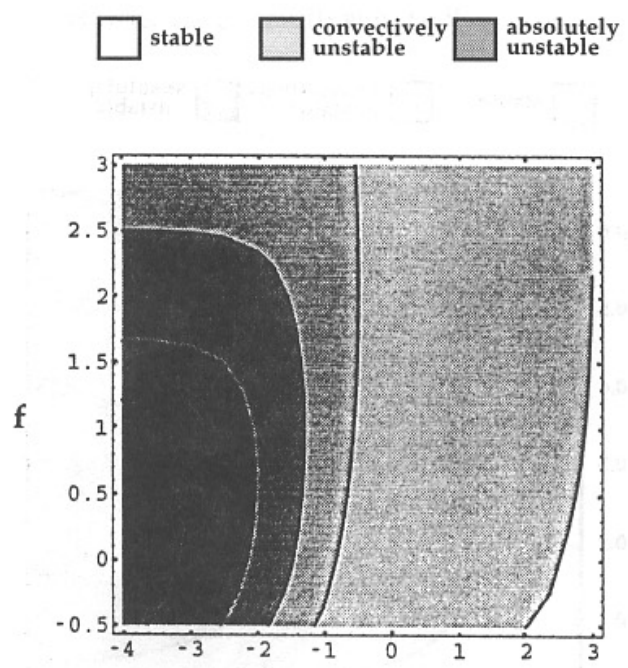

(b)

$\log$ a

FIG. 2. (a) Domains of absolute and convective instabilities in the $(a, c)$ plane. (b) Isolines of the threshold value of $c$ as a function of $a$ and $f$.

beds, the convective/absolute transition condition $d \Omega / d K=0$ with $\Omega_{i}^{0}=0$ is written as

$-2 a+\left[2 f c^{2}+c(1-f)\right] \cdot\left[4 a+4 f c^{2}+c^{2}(1-f)^{2}\right]=0$

and the absolute frequency which is real at threshold is

$$
\Omega_{r}^{0}=\left\{\frac{2 f c^{2}+c(1-f)}{2 a}\right\}^{1 / 2} .
$$

The domains of absolute and convective instabilities are shown in the $(a, c)$ plane in Fig. 2(a) for $f=1$. The isolines of the threshold value of $c$ are also plotted as a function of $a$ and $f$ in Fig. 2(b). The variation of the threshold value of $c$, which characterizes the onset of the absolute instability, depends weakly upon the dissymmetry parameter $f$.

\section{Properties of the dominant waves}

Since the onset of the absolute instability depends weakly upon $f$, the parameter $f$ has been set to unity in the following discussion. In this case, the convective/absolute transition corresponds to the threshold value $c_{A}=\left\{\left[\left(a^{2}+a\right)^{1 / 2}-a\right] / 2\right\}^{1 / 2}$. The absolute growth rate $\Omega_{i}^{0}$ is positive for $c<c_{A}$. This condition defines the absolutely un-
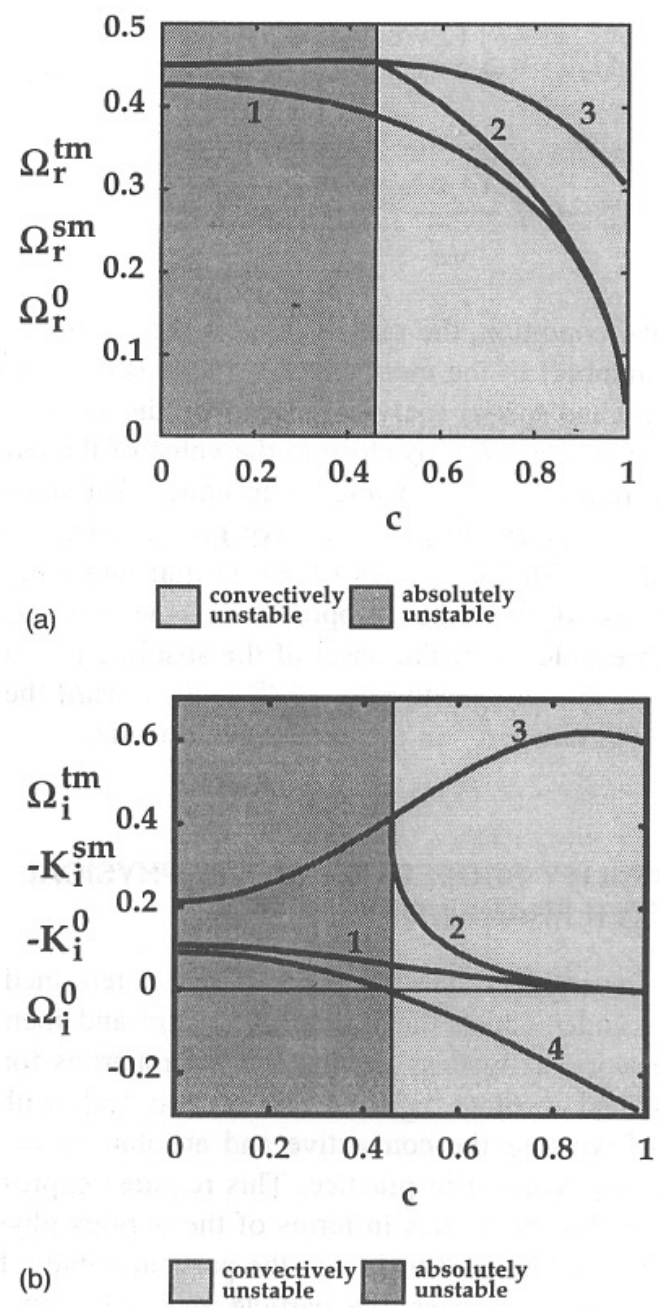

FIG. 3. (a) Effect of the coefficient $c$ on the most temporally unstable frequency $\Omega_{r}^{t m}(1)$, the most spatially unstable frequency $\Omega_{r}^{s m}(2)$, and the absolute frequency $\Omega_{r}^{0}$ (3) with $a=f=1$. (b) Effect of the coefficient $c$ on the temporal maximum growth rate $\Omega_{i}^{t m}(1)$, the spatial maximum growth rate $-K_{i}^{s m}(2)$, the spatial absolute growth rate $-K_{i}^{0}(3)$, and the temporal absolute growth rate $\Omega_{i}^{0}$ (4) with $a=f=1$.

stable domain. In order to compare the results of the temporal and spatial analyses, the viscous parameter $a$ is set equal to unity for convenience, and $c$ remains the only free parameter. Other values give the same qualitative behavior. Figures 3(a) and 3(b) show the dependencies on $c$ of the frequencies and the wave numbers of the most unstable temporal wave $\left(K_{r}^{t m}, \Omega_{r}^{t m}+i \Omega_{i}^{t m}\right)$, the most unstable spatial wave $\left(K_{r}^{s m}+i K_{i}^{s m}, \Omega_{r}^{s m}\right)$, and the absolute wave $\left(K_{r}^{0}+i K_{i}^{0}\right.$, $\left.\Omega_{r}^{0}+i \Omega_{i}^{0}\right)$. It should be mentioned that the maximum spatial growth rate $-K_{i}^{s m}$ and the corresponding frequency $\Omega_{r}^{s m}$ are only defined in the convective domain $\left(c>c_{A}\right)$.

These frequencies and wave numbers have been investigated in the neighborhood of the critical condition $(c=1)$. Asymptotic analysis for $K_{r} \sim 0$ gives the following scalings:

$$
\Omega_{r}^{s m} \sim \Omega_{r}^{t m} \sim 0.7 \frac{\sqrt{1-c}}{\sqrt{a}},
$$




$$
\begin{aligned}
& -K_{i}^{s m} \sim \Omega_{i}^{t m} \sim 0.5 \frac{(1-c)^{2}}{a}, \\
& K_{r}^{s m} \sim K_{r}^{t m} \sim 0.7 \frac{\sqrt{1-c}}{\sqrt{a}} .
\end{aligned}
$$

Near critical condition, the same value for the frequency (or the wave number) of the most unstable mode is obtained by the temporal and spatial analyses. Moreover, the value of the spatial growth rate $-K_{i}^{s m}$ is close to the value of the temporal growth rate $\Omega_{i}^{t m}$. It is of interest to notice that since the growth rates vary as $(1-c)^{2}$, the wave grows rather slowly near the threshold. The values of the spatial and temporal growth rates differ when approaching the convective/ absolute threshold. Near the onset of the absolute instability $\left(c=c_{A}\right)$, the spatial growth rate $-K_{i}^{s m}$ tends toward the absolute growth rate $-K_{i}^{0}$ in the convective domain.

\section{INSTABILITY PROPERTIES IN THE PHYSICAL PARAMETER FRAMEWORK}

The above linear stability analysis has determined the conditions under which the instability occurs and then becomes absolute, as well as the instability properties for the convective and absolute regimes. This section deals with the question of whether the convective and absolute instability conditions are realized in practice. This requires expression of the instability properties in terms of the various physical parameters of a fluidized bed, e.g., the particle volume fraction, the particle diameter, the particle and fluid densities, etc. Since the instability properties have been expressed in terms of the dimensionless coefficients, $a, c$, and $f$, it is thus necessary to formulate these coefficients in terms of the physical parameters through the use of the theories of Batchelor ${ }^{13}$ and Anderson and Jackson. ${ }^{18,26}$ Since some of the quantities in both models are difficult to measure experimentally or to compute theoretically, only rough estimates are given by these authors. Therefore, the results given in this section depend upon the specific relations for these quantities, i.e., the elastic term, the added mass term, the particle viscosity and drag, etc., that are given by the theories. Improved estimates of these quantities will yield more precise computations of the instability properties.

\section{A. Batchelor's theory}

The case of a gas-fluidized bed in which the fluid is air at normal temperature and pressure is considered first. Figure 4(a) shows the domains of absolute and convective instabilities in the plane $\left(d_{s}, \phi_{0}\right)$ with the rest of the parameters kept constant ( $\phi_{0}$ is the mean particle volume fraction). The values of the parameters are given in Table I and correspond to those given by Batchelor ${ }^{13}$ in his Sec. V. It should be mentioned that, as indicated by Batchelor, ${ }^{13}$ the particle viscosity $\eta$, the elastic coefficient $Q$, and the Richardson-Zaki index $n$ are regarded as invariant with $\phi_{0}$ and the virtual mass effects are negligibly small in this case. The marginal stability curve displayed in Fig. 4(a) corresponds exactly to the

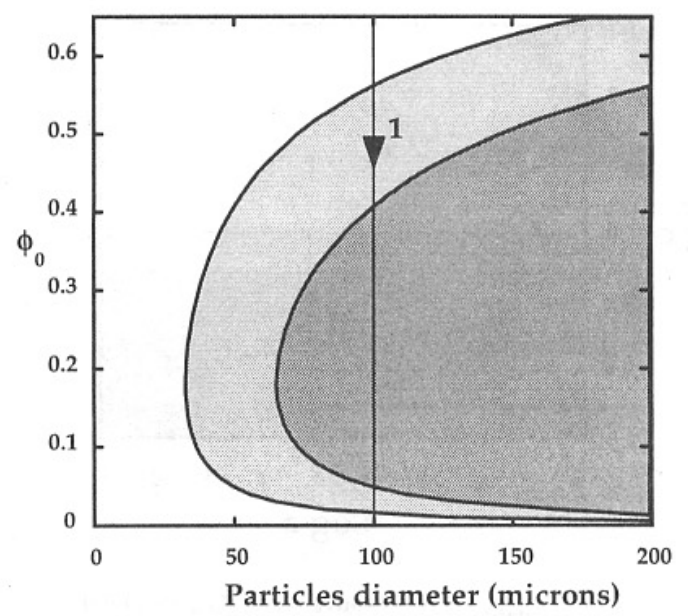

(a)
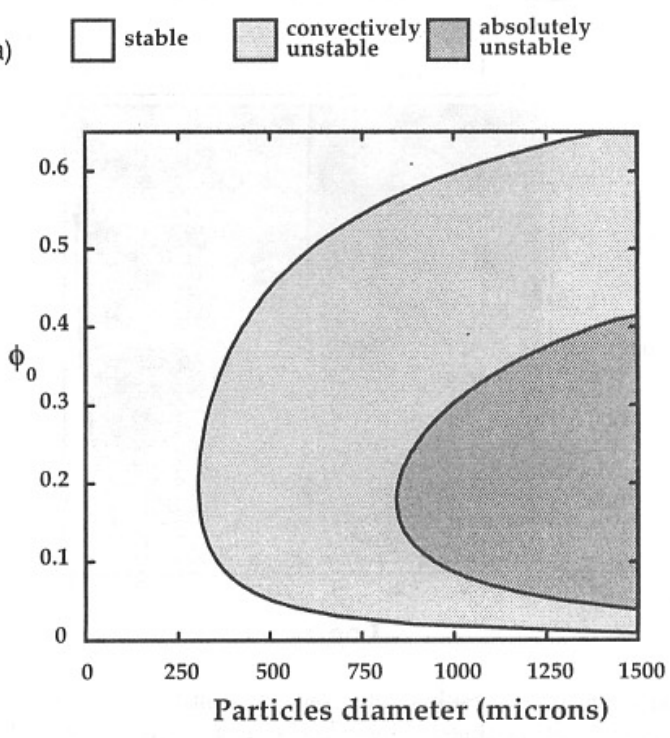

(b)

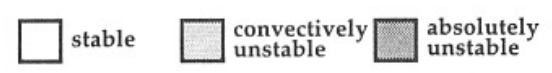

FIG. 4. (a) Instability domains for a gas-fluidized bed from Batchelor's theory. (b) Instability domains for a liquid-fluidized bed from Batchelor's theory.

stability conditions shown in Fig. 3 of Batchelor. ${ }^{13}$ It should be noted that the stable region decreases with increasing particle diameter.

Consider the behavior of the flow for a fixed particle diameter, for instance $d_{s}=100 \mu \mathrm{m}$ [trajectory 1 displayed in Fig. 4(a)]. When the flow rate is large enough to fluidize the bed, $\phi_{0}$ is close to its maximum value and the flow is stable. As the flow rate is increased, $\phi_{0}$ decreases and the flow can become convectively unstable and then absolutely unstable. The flow becomes convectively unstable and then stable again at even higher flow rates and smaller values of $\phi_{0}$.

The evolution of the spatial branches along the trajectory 1 of Fig. 4(a) is displayed in Fig. 5. At the convective/ absolute transition, a saddle point appears. The branch switching corresponds to the disappearance of the spatially amplified wave and to the outbreak of the absolute instability. It should be noted that the absolute frequency is surpris- 
TABLE I. Values of the parameters given by Batchelor (Ref. 13) for gas-fluidized and liquid-fluidized beds.

\begin{tabular}{|c|c|c|c|c|c|c|c|c|c|}
\hline Parameter & $\begin{array}{c}\rho_{s} \\
\left(\mathrm{~g} \mathrm{~cm}^{-3}\right)\end{array}$ & $\begin{array}{c}\rho_{f} \\
\left(\mathrm{~g} \mathrm{~cm}^{-3}\right)\end{array}$ & $\begin{array}{c}\eta_{f} \\
\left(\mathrm{~g} \mathrm{~cm}^{-1} \mathrm{~s}^{-1}\right)\end{array}$ & $\begin{array}{c}U_{0} \\
\left(\mathrm{~cm} \mathrm{~s}^{-1}\right)\end{array}$ & $n$ & $\gamma$ & $\frac{Q}{\left(\mathrm{~cm}^{2} \mathrm{~s}^{-2}\right)}$ & $\begin{array}{c}\eta \\
\left(\mathrm{cm}^{2} \mathrm{~s}^{-1}\right)\end{array}$ & $C(\phi)$ \\
\hline $\begin{array}{l}\text { Gas-fluidized } \\
\text { bed }\end{array}$ & 1 & $1.29 \times 10^{-3}$ & $1.85 \times 10^{-4}$ & $\frac{g\left(\rho_{s}-\rho_{f}\right) d_{s}^{2}}{18 \eta_{f}}$ & 5.5 & 1 & $\begin{array}{c}\alpha \frac{d_{s}}{2} \gamma g \frac{\rho_{s}-\rho_{f}}{\rho_{s}}, \\
\text { with } \alpha=1\end{array}$ & $\begin{array}{c}\beta\left(d_{s} / 2\right) U(\phi), \\
\text { with } \beta=1\end{array}$ & 0 \\
\hline $\begin{array}{l}\text { Liquid-fluidized } \\
\text { bed }\end{array}$ & 2.5 & 1 & $10^{-2}$ & $\frac{g\left(\rho_{s}-\rho_{f}\right) d_{s}^{2}}{18 \eta_{f}}$ & 5.5 & $1+\frac{0.63 \sqrt{R_{e}}}{4.9+0.63 \sqrt{R_{e}}}$ & $\begin{array}{c}\alpha \frac{d_{s}}{2} \gamma g \frac{\rho_{s}-\rho_{f}}{\rho_{s}}, \\
\text { with } \alpha=1\end{array}$ & $\begin{array}{c}\beta\left(d_{s} / 2\right) U(\phi), \\
\text { with } \beta=1\end{array}$ & $\frac{1+2 \phi}{2(1-\phi)}$ \\
\hline
\end{tabular}

ingly large $(\approx 80 \mathrm{~Hz})$ for the set of parameters chosen by Batchelor. $^{13}$

The same qualitative behavior is obtained for a liquidfluidized bed where the fluid is water, as displayed in Fig. 4(b). The values of the parameters given by Batchelor ${ }^{13}$ in his Sec. V are indicated in Table I. Again, the particle viscosity $\eta$, the elastic coefficient $Q$, and the Richardson-Zaki index $n$ are supposed constant with $\phi_{0}$. The expression for the virtual mass function $C(\phi)$ is that suggested by Zuber. ${ }^{34}$ The drag-slope parameter $\gamma$ is defined by Eq. (3.3) of Batchelor with the empirical expression for the drag coefficient $C_{D}=\left(0.63+4.90 \mathrm{Re}^{-1 / 2}\right)^{2}$ [Eq. (5.27) of Batchelor]. It should be noticed that the convectively unstable region is larger for a liquid-fluidized bed than that for a gas-fluidized bed.

\section{B. Anderson and Jackson's theory}

The same type of analysis has been conducted with the use of Anderson and Jackson's theory. ${ }^{18}$ The case of a bed of beads fluidized by water is only considered in this case since estimates of the parameters inferred from the comparison with experimental observations are available in this case. Figure 6 shows the domains of absolute and convective instabilities in the plane $\left(d p^{s} / d \phi, \phi_{0}\right)$ with the rest of the parameters kept constant. The values of the parameters given

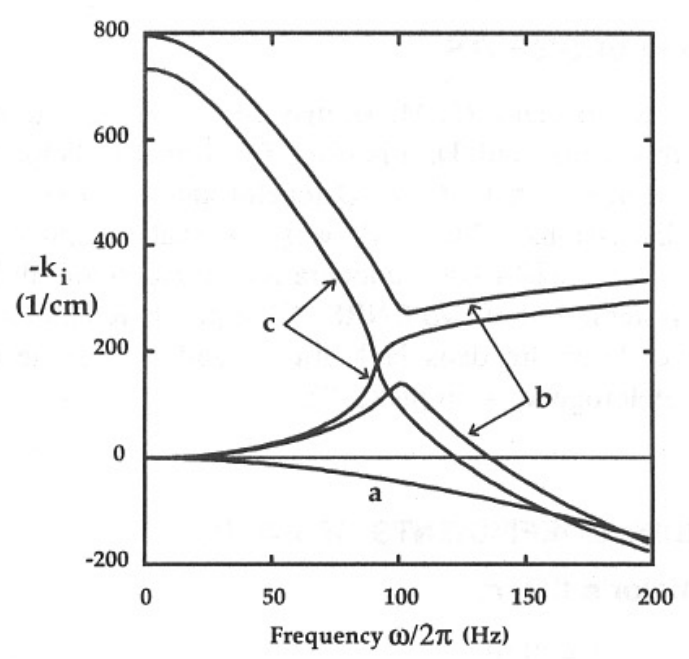

FIG. 5. Effect of the decrease of the particle volume fraction on the spatial growth rate of the wave from Batchelor's theory: $\phi_{0}=0.60$ (a), $\phi_{0}=0.42$ (b), $\phi_{0}=0.40$ (c). The particle diameter is $d_{s}=100 \mu \mathrm{m}$ and the fluidizing fluid is air. by Anderson and Jackson ${ }^{18}$ in their Table I are indicated here in Table II. The particle bulk and shear viscosities $\lambda^{s}$ and $\mu^{s}$ and the Richardson-Zaki index $n$ are supposed independent of $\phi_{0}$, because of lack of information. The value of the virtual mass $C(\phi)$ coefficient is $1 / 2$ which corresponds to that of an isolated sphere. The "dead zone" indicated in Fig. 6 corresponds to a set of parameters for which no solutions can be found for the dispersion relation and thus to an unphysical set of parameters.

Since $d p^{s} / d \phi$ represents an elastic behavior of the particle phase, it measures the stabilizing parameter. For a small value of $d p^{s} / d \phi$ (trajectory 1 displayed in Fig. 6), when the flow rate is increased enough to fluidize the bed, $\phi_{0}$ is large but the flow is always convectively unstable. As the flow rate is increased, $\phi_{0}$ decreases and the flow can become absolutely unstable. The flow becomes convectively unstable and then stable at larger flows rate and smaller values of $\phi_{0}$. Conversely, for a larger value of $d p^{s} / d \phi$ (trajectory 2 displayed in Fig. 2), the flow is first stable at large values of $\phi_{0}$. When the flow rate is increased, the flow becomes convectively unstable and then stable again. In this case, no absolutely unstable region is accessible. For even larger values of

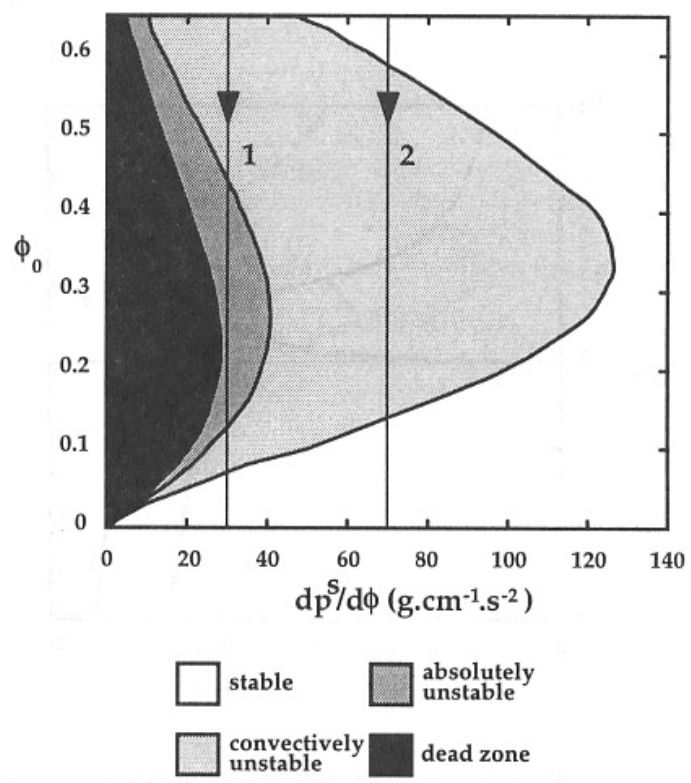

FIG. 6. Instability domains for a liquid-fluidized bed from Anderson and Jackson's theory. 


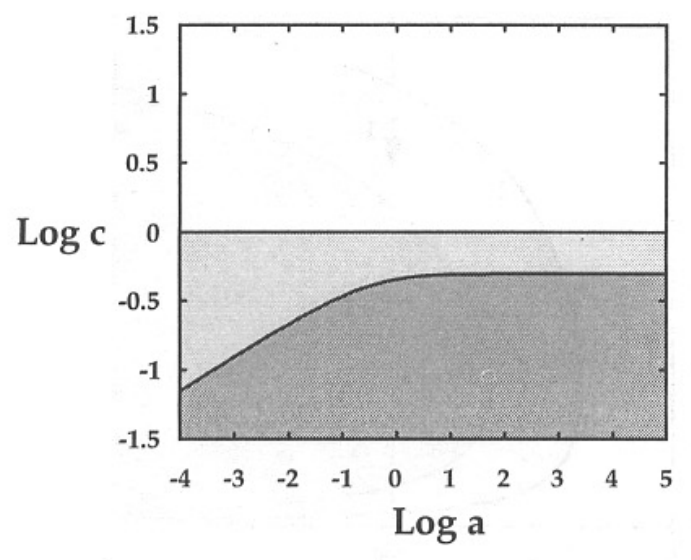

(a)
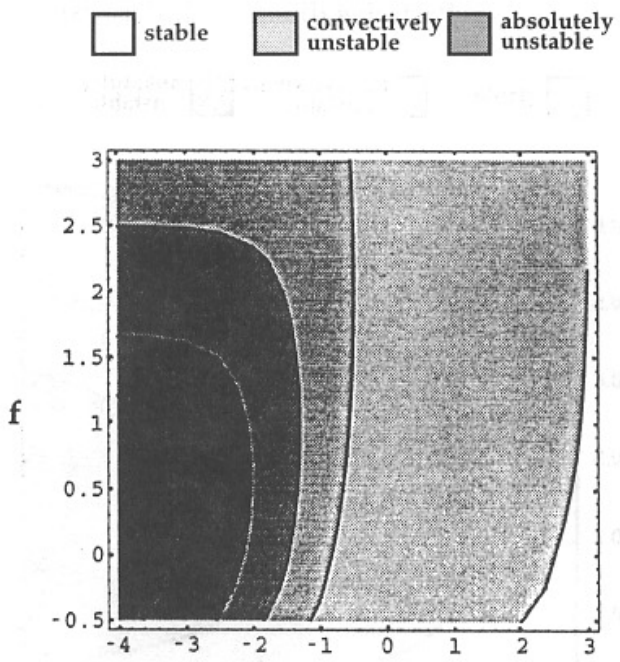

(b)

$\log a$

FIG. 2. (a) Domains of absolute and convective instabilities in the $(a, c)$ plane. (b) Isolines of the threshold value of $c$ as a function of $a$ and $f$.

beds, the convective/absolute transition condition $d \Omega / d K=0$ with $\Omega_{i}^{0}=0$ is written as

$-2 a+\left[2 f c^{2}+c(1-f)\right] \cdot\left[4 a+4 f c^{2}+c^{2}(1-f)^{2}\right]=0$

and the absolute frequency which is real at threshold is

$$
\Omega_{r}^{0}=\left\{\frac{2 f c^{2}+c(1-f)}{2 a}\right\}^{1 / 2} .
$$

The domains of absolute and convective instabilities are shown in the $(a, c)$ plane in Fig. 2(a) for $f=1$. The isolines of the threshold value of $c$ are also plotted as a function of $a$ and $f$ in Fig. 2(b). The variation of the threshold value of $c$, which characterizes the onset of the absolute instability, depends weakly upon the dissymmetry parameter $f$.

\section{Properties of the dominant waves}

Since the onset of the absolute instability depends weakly upon $f$, the parameter $f$ has been set to unity in the following discussion. In this case, the convective/absolute transition corresponds to the threshold value $c_{A}=\left\{\left[\left(a^{2}+a\right)^{1 / 2}-a\right] / 2\right\}^{1 / 2}$. The absolute growth rate $\Omega_{i}^{0}$ is positive for $c<c_{A}$. This condition defines the absolutely un-

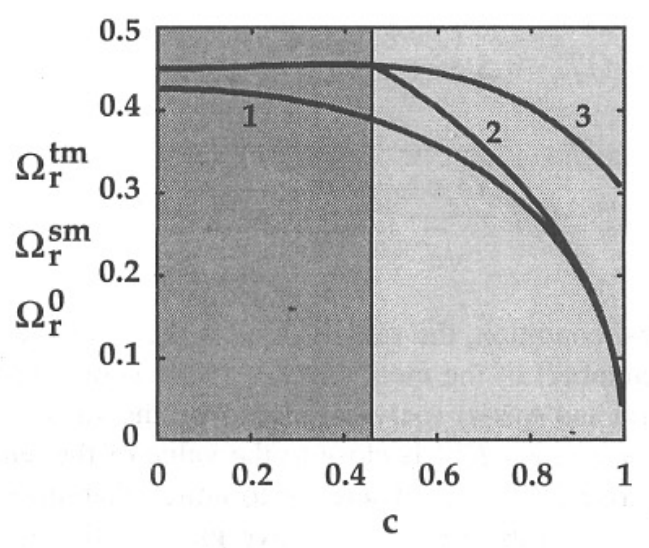

(a)
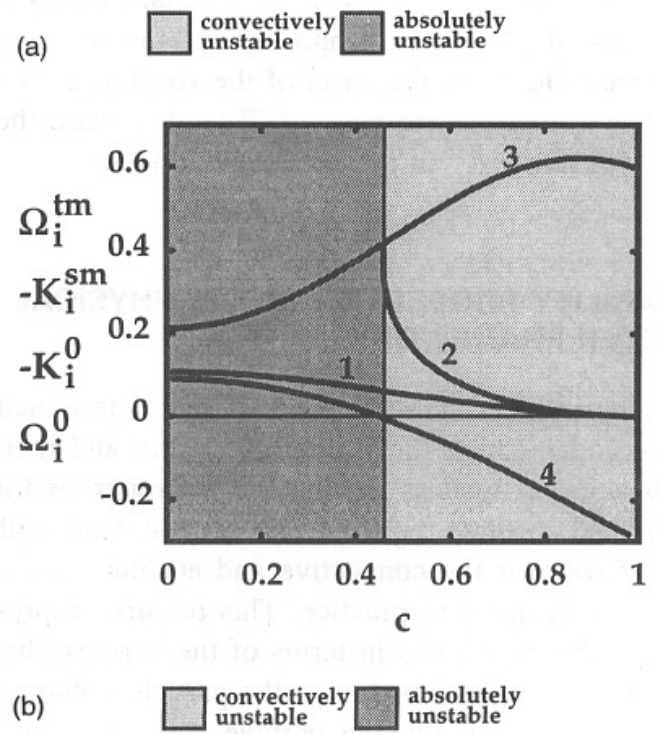

FIG. 3. (a) Effect of the coefficient $c$ on the most temporally unstable frequency $\Omega_{r}^{t m}(1)$, the most spatially unstable frequency $\Omega_{r}^{s m}(2)$, and the absolute frequency $\Omega_{r}^{0}$ (3) with $a=f=1$. (b) Effect of the coefficient $c$ on the temporal maximum growth rate $\Omega_{i}^{t m}(1)$, the spatial maximum growth rate $-K_{i}^{s m}(2)$, the spatial absolute growth rate $-K_{i}^{0}(3)$, and the temporal absolute growth rate $\Omega_{i}^{0}$ (4) with $a=f=1$.

stable domain. In order to compare the results of the temporal and spatial analyses, the viscous parameter $a$ is set equal to unity for convenience, and $c$ remains the only free parameter. Other values give the same qualitative behavior. Figures 3(a) and 3(b) show the dependencies on $c$ of the frequencies and the wave numbers of the most unstable temporal wave $\left(K_{r}^{t m}, \Omega_{r}^{t m}+i \Omega_{i}^{t m}\right)$, the most unstable spatial wave $\left(K_{r}^{s m}+i K_{i}^{s m}, \Omega_{r}^{s m}\right)$, and the absolute wave $\left(K_{r}^{0}+i K_{i}^{0}\right.$, $\left.\Omega_{r}^{0}+i \Omega_{i}^{0}\right)$. It should be mentioned that the maximum spatial growth rate $-K_{i}^{s m}$ and the corresponding frequency $\Omega_{r}^{s m}$ are only defined in the convective domain $\left(c>c_{A}\right)$.

These frequencies and wave numbers have been investigated in the neighborhood of the critical condition $(c=1)$. Asymptotic analysis for $K_{r} \sim 0$ gives the following scalings:

$$
\Omega_{r}^{s m} \sim \Omega_{r}^{t m} \sim 0.7 \frac{\sqrt{1-c}}{\sqrt{a}},
$$




$$
\begin{aligned}
& c_{1} \cdot c_{2}=-\frac{Q}{1+\theta(\phi)}, \\
& v=\frac{\eta}{1+\theta(\phi)}
\end{aligned}
$$

with $\theta(\phi)=\left(\rho_{f} / \rho_{s}\right) C(\phi), \quad \zeta(\phi)=\left(\rho_{f} / \rho_{s}\right) \phi(d C / d \phi)$, and where $\gamma$ is the drag-slope parameter defined by Eq. (3.3) of Batchelor. $^{13}$

\section{Anderson and Jackson's theory}

$$
\begin{aligned}
& \tau=\left[1+\frac{\rho_{s}}{\rho_{f}} \frac{1-\phi}{\phi}+\frac{C(\phi)}{\phi(1-\phi)}\right] \frac{\rho_{f}}{\rho_{s}-\rho_{f}} \frac{U}{g} \frac{\phi}{1-\phi}, \\
& c_{1}+c_{2}=\frac{2[1+C(\phi) /(1-\phi)][U /(1-\phi)]}{1+\left[\left(\rho_{s} / \rho_{f}\right)(1-\phi) / \phi\right]+\{C(\phi) /[\phi(1-\phi)]\}}, \\
& c_{1} \cdot c_{2}=\frac{[1+C(\phi) /(1-\phi)][U /(1-\phi)]^{2}-\left[\left(1 / \rho_{f}\right)\left(d p^{s} / d \phi\right)(1-\phi) / \phi\right]}{1+\left[\left(\rho_{s} / \rho_{f}\right)(1-\phi) / \phi\right]+\{C(\phi) /[\phi(1-\phi)]\}}, \\
& v=\frac{\left[\left(\lambda_{0}^{s}+4 / 3 \mu_{0}^{s}\right) / \rho_{f}\right]\left[(1-\phi) / \phi^{2}\right]}{1+\left[\left(\rho_{s} / \rho_{f}\right)(1-\phi) / \phi\right]+\{C(\phi) /[\phi(1-\phi)]\}} .
\end{aligned}
$$

${ }^{1}$ J. F. Davidson, R. Clift, and D. Harrison, Fluidization (Academic, London, 1985).

${ }^{2}$ J. M. Ham, S. Thomas, E. Guazzelli, G. M. Homsy, and M.-C. Anselmet, "An experimental study of the stability of liquid-fluidized beds," Int. J. Multiphase Flow 16, 171 (1990).

${ }^{3} \mathrm{~S}$. C. Tsinontides and R. Jackson, "The mechanics of gas-fluidized beds with an interval of stable fluidization," J. Fluid Mech. 255, 237 (1993).

${ }^{4}$ R. Clift and J. R. Grace, "Continuous bubbling and slugging," in Fluidization, edited by J. F. Davidson, R. Clift, and D. Harrison (Academic, London, 1985), p. 73.

${ }^{5}$ J. F. Davidson, D. Harrison, and J. R. F. Guedes de Carvalho, "On the liquid-like behavior of fluidized beds," Annu. Rev. Fluid Mech. 9, 55 (1977).

${ }^{6}$ T. B. Anderson and R. Jackson, "A fluid mechanical description of fluidized beds-Comparison of theory and experiment," Ind. Eng. Chem. Fundam. 8, 137 (1969).

${ }^{7}$ M. M. El-Kaissy and G. M. Homsy, "Instability waves and the origin of bubbles in fluidized beds. Part I. Experiments," Int. J. Multiphase Flow 2, 379 (1976).

${ }^{8}$ A. K. Didwania and G. M. Homsy, "Flow regime and flow transitions in liquid-fluidized beds," Int. J. Multiphase Flow 7, 563 (1981).

${ }^{9}$ A. K. Didwania and G. M. Homsy, "Resonant sideband instabilities in wave propagation in fluidized beds," J. Fluid Mech. 122, 433 (1982).

${ }^{10} \mathrm{G}$. K. Batchelor, "Secondary instability of a gas-fluidized bed," J. Fluid Mech. 257, 359 (1993).

${ }^{11}$ R. Jackson, "Hydrodynamic stability of fluid particle systems," in Fluidization, edited by J. F. Davidson, R. Clift, and D. Harrison (Academic, London, 1985), p. 47.

${ }^{12} \mathrm{G}$. M. Homsy, "Aspects of flow and mixing behavior in fluidized beds," in Disorder and Mixing, edited by E. Guyon, J.-P. Nadal, and Y. Pomeau (Kluwer Academic, Dordrecht, 1985), p. 185.

${ }^{13} \mathrm{G}$. K. Batchelor, "A new theory of the instability of a uniform fluidized bed," J. Fluid Mech. 193, 75 (1988).

${ }^{14}$ G. K. Batchelor and J. M. Nitsche, "Instability of stationary unbounded stratified fluid," J. Fluid Mech. 227, 357 (1991).

${ }^{15}$ J. D. Murray, "On the mathematics of fluidization. I," J. Fluid Mech. 21, 465 (1965).

${ }^{16}$ R. L. Pigford and T. Baron, "Hydrodynamic stability of fluidized bed," Ind. Eng. Chem. Fundam. 1, 81 (1965).
${ }^{17} \mathrm{O}$. Molerus, "Hydrodynamische stabilitat des fliessbetts," Chem. Ing. Technik. 39, 341 (1967).

${ }^{18} \mathrm{~T}$. B. Anderson and R. Jackson, "A fluid mechanical description of fluidized beds-Stability of the state of uniform fluidization," Ind. Eng. Chem. Fundam. 7, 12 (1968).

${ }^{19}$ S. K. Garg and J. W. Pritchett, "Dynamics of gas-fluidized beds," J. Appl. Phys. 46, 4493 (1975).

${ }^{20}$ G. M. Homsy, M. M. El-Kaissy, and A. K. Didwania, "Instability waves and the origin of bubbles in fluidized beds. Part II. Comparison with theory," Int. J. Multiphase Flow 6, 305 (1980).

${ }^{21}$ D. J. Needham and J. H. Merkin, "The propagation of voidage disturbance in a uniformly fluidized bed," J. Fluid Mech. 131, 427 (1983).

${ }^{22}$ M. F. Göz, "On the origin of wave patterns in fluidized beds," J. Fluid Mech. 240, 379 (1992).

${ }^{23} \mathrm{R}$. J. Briggs, Electron-Stream Interaction with Plasmas (MIT Press, Cambridge, MA, 1964).

${ }^{24} \mathrm{~A}$. Bers, "Linear waves and instabilities," in Physics of Plasmas, edited by C. de Witt and J. Peyraud (Gordon and Breach, New York, 1975), p. 117.

${ }^{25}$ P. Huerre and P. A. Monkewitz, "Local and global instabilities in spatially developing flows," Annu. Rev. Fluid Mech. 22, 473 (1990).

${ }^{26} \mathrm{~T}$. B. Anderson and R. Jackson, "A fluid mechanical description of fluidized beds-Equation of motion," Ind. Eng. Chem. Fundam. 6, 527 (1967).

${ }^{27} \mathrm{R}$. Jackson, "The mechanic of fluidized bed," Trans. Inst. Chem. Eng. 41, 13 (1963).

${ }^{28}$ G. B. Whitham, Linear and Nonlinear Waves (Wiley-Interscience, New York, 1974).

${ }^{29}$ J. T. C. Liu, "Note on a wave-hierarchy interpretation of fluidized bed instabilities," Proc. R. Soc. London Ser. A 380, 229 (1982).

${ }^{30}$ G. J. Kynch, "A theory of sedimentation," Trans. Faraday Soc. 48, 166 (1952).

${ }^{31}$ J. F. Richardson and W. N. Zaki, "Sedimentation and fluidization: Part I," Trans. Inst. Chem. Eng. 32, 35 (1954).

${ }^{32}$ G. B. Wallis, One-Dimensional Two-Phase Flow (McGraw-Hill, New York, 1969).

${ }^{33}$ J. M. Chomaz, P. Huerre, and L. Redekopp, "A frequency selection criterion in spatially developing flows," Stud. Appl. Math. 84, 119 (1991).

${ }^{34} \mathrm{~N}$. Zuber, "On the dispersed two-phase flow in the laminar flow regime," Chem. Eng. Sci. 19, 897 (1964). 